Introduction The pathogenesis of Inflammatory Bowel Disease (IBD) is unclear which hinders effective targeted drug development. IBD and murine models of colitis are associated with the abnormal accumulation of activated dendritic cells (DCs) in the colonic epithelium. DCs play a critical role in promoting inflammatory responses and blockade of their activation prevents colitis development in mouse models. We now propose to address the mechanisms underlying the aberrant accumulation of DCs in the gut by focusing on microbial danger stimuli that drive activation and migration of DCs. Methods We analysed the expression of migration-associated markers on DCs from normal and colitic mice. Bone marrow derived DCs (BMDCs) from WT or Beta-2 Integrin ${ }^{-/}\left(\right.$ITGB2 $\left.^{-/}\right)$mice were cultured in vitro and their migration and activation analysed in response to control (Phosphate buffered saline - PBS), bacterial lipopolysaccharide (LPS), live Escherichia coli (EC), and live Bacteroides fragilis $(\mathrm{BF})$ in the presence and absence of the lamina propria extracellular matrix component fibronectin.

Results During colitis there was a marked increase in a population of CD103- ( $a_{\mathrm{E}}$ integrin) DCs. We were able to mirror these populations in vitro. DCs moved via random motion and their velocity after stimulation with LPS and EC, in the absence of fibronectin, was significantly decreased. In comparison, stimulation with BF significantly increased DC velocity ( $p<0.001$ for all). In the presence of fibronectin, there was no change in DC velocity. The track displacement length (the distance between the start and finishing point of a given cell migration track) was significantly decreased after EC stimulation and significantly increased after BF stimulation ( $p<0.01$ for both). Unstimulated ITGB2 $\%$ BMDC velocity and track displacement length were significantly increased in comparison to that of unstimulated WT BMDC ( $p<0.001$ for both). This was more marked in the absence of fibronectin.

Conclusion We have shown that in vitro WT DC cultures contain DCs with similar integrin-defined phenotypes to those found in colonic DCs in colitis. Differential bacterial stimulation causes opposing fibronectin-dependent effects on BMDC migratory behaviour whilst absence of ITGB2 significantly alters the migratory behaviour of BMDC. Our data implicates a complex relationship between specific components of gut microbiota, extracellular matrix, and migration and activation of DCs that could potentiate the aberrant accumulation of DCs in the colitic gut. If this complex relationship is further elucidated, it may be possible in the future to develop therapies that reduce colitis by controlling DC migration. Disclosure of Interest None Declared.

\section{PTH-104 THE ROUTINE MEASUREMENT OF THIOPURINE METABOLITE LEVELS RESULTS IN DOSE OPTIMISATION IN ONE THIRD OF IBD PATIENTS; RESULTS FROM A DISTRICT GENERAL HOSPITAL}

doi:10.1136/gutjnl-2013-304907.591

${ }^{1} \mathrm{H}$ M Dewhurst, ${ }^{2} \mathrm{H}$ E Johnson, ${ }^{3} \mathrm{~J}$ Begley, ${ }^{2} \mathrm{~S}$ A Weaver, ${ }^{2,}$ S D McLaughlin. ${ }^{1}$ Research; ${ }^{2}$ Gastroenterology; ${ }^{3}$ Clinical Biochemistry, Royal Bournemouth Hospital, Bournemouth, UK

Introduction Measuring azathioprine or mercaptopurine (AZA) metabolite levels 6-TGN and 6-MMPN allows identification of patients who are: 1 . Non compliant with their medication, 2. On a sub-optimal doe, 3. On a supra-therapeutic dose, 4. Are preferentially metabolising azathioprine to methylated metabolites (6-MMPN:6-TGN ratio > 11).

Our own and others published data demonstrate that measuring metabolite levels in patients failing azathioprine therapy followed by appropriate changes in dosing and/or the addition of allopurinol (with $75 \%$ dose reduction in AZA) can result in clinical remission in the majority of patients 1 . We report the outcome of the routine measurement of metabolite levels in patients treated with AZA who were in a clinical remission without side effects or abnormal liver function tests (LFTs).

Methods All patients underwent TPMT testing, azathioprine and mercaptopurine were initiated at doses of $2 \mathrm{mg} / \mathrm{kg}$ and $1 \mathrm{mg} / \mathrm{kg}$ respectively in those with wild-type TPMT with a $50 \%$ reduction in dose in TPMT heterozygotes. We searched the prospective database maintained by our biochemistry department for all patients who underwent metabolite level testing from September 2011 to November 2012, hospital case notes for these patients were reviewed. The indications, results of testing, changes in clinical management and patient outcomes were recorded.

Results 108 patients underwent metabolite testing, median length of follow-up since testing was 287.6 days (range 21-441), all were stable on AZA for $>4$ weeks with normal LFTs and in a clinical remission. 38 (35.2\%) had UC, 66 (61.1\%) CD, 52 (48.1\%) were male.

$17(15.7 \%)$ patients had a sub-theraputic 6-TGN, 10 (9.3\%) supra-theraputic 6-TGN level $(>800)$ all of whom had dose optimisation. $6(5.6 \%)$ patients were hypermethylators these were switched to allopurinol co-therapy with an appropriate reduction in AZA dose.

Conclusion In the present study the routine measurement of AZA metabolites resulted in a change in clinical management in $30.6 \%$ of patients.

Whilst unproven in prospective longitudinal studies logic suggests that the routine measurement of AZA metabolites in all patients commenced on thiopurines followed by appropriate dose optimisation (with or without allopurinol co-prescription) should reduce or prevent the development of drug side effects, abnormal LFTs and bone marrow suppression and reduce the risk of disease relapse. We recommend that AZA metabolite testing is performed in all patients 4-6 weeks after commencing AZA.

Disclosure of Interest None Declared.

\section{PTH-105 THE EFFECT OF INFLIXIMAB PRE-TREATED HUMAN BLOOD- ENRICHED DENDRITIC CELLS FROM PATIENTS WITH ACTIVE CROHN'S DISEASE AND HEALTHY CONTROLS ON SUBSEOUENT HUMAN T-LYMPHOCYTE PHENOTYPE AND CYTOKINE PRODUCTION IN VITRO}

doi:10.1136/gutjnl-2013-304907.592

1. S T C Peake, ${ }^{2} \mathrm{D}$ Bernardo, ${ }^{2} \mathrm{E}$ Mann, ${ }^{1} \mathrm{~J}$ Landy, ${ }^{2} \mathrm{H}$ Omar, ${ }^{2} \mathrm{~S}$ C Knight, ${ }^{1} \mathrm{~A}$ L Hart. ${ }^{1} / B D$ Unit, St Mark's Hospital; ${ }^{2} A P R G$, Imperial College, London, UK

Introduction Dendritic cells (DC) play a key role in discriminating between commensal microorganisms and potentially harmful pathogens. Expression of surface markers and cytokine production by DC at the time of antigen presentation control T-cell differentiation, cytokine profile \& homing properties imprinted on stimulated T-cells. This process defines the type of immune response that occurs and its anatomical location. In $\mathrm{CD}$, dysregulation of the immune response to gut microbiota and aberrant immune cell trafficking play a central role in disease pathogenesis. Infliximab (IFX) is an effective treatment for CD, but its mechanism of action is unclear. In this study, we investigated the effect of IFX pre-treated blood-enriched DC, isolated from patients with active CD and healthy controls (HC), on human T-cell proliferation, phenotype\&cytokine production

Methods Low density cells (LDC), enriched for DC, were obtained following Ficoll and Nycoprep gradient separation of fresh blood from patients with active ileocolonic CD (CDAI > 220) and HC. LDC were cultured $\left(0.5 \times 10^{6} \mathrm{cells} / \mathrm{ml}\right)$ with IFX $(1.10 \mu \mathrm{g} \& 100 \mu \mathrm{g} /$ $\mathrm{ml}$ \&basal) for $24 \mathrm{hr}$. T-cells were enriched from allogeneic $\mathrm{HC}$ blood and labelled with CFSE. LDC were added to T-cells in complete medium $(400,000$ cells $/ \mathrm{ml})$ at basal,1.2\&3\% concentrations and incubated for 5 days. Following incubation, T-cell proliferation, 